\title{
LOWER AND UPPER BOUNDS FOR COHEN-MACAULAY DIMENSION
}

\section{J. Asadollahi and Sh. Salarian}

\begin{abstract}
Lower and upper bounds for $\mathrm{CM}$-dimension, called $\mathrm{CM}_{*}$-dimension and $\mathrm{CM}^{*}$-dimension, will be defined for any finitely generated module $M$ over a local Noetherian ring $R$. Both $\mathrm{CM}_{*}$ and $\mathrm{CM}^{*}$-dimension reflect the Cohen-Macaulay property of rings. Our results will show that these dimensions have the expected basic properties parallel to those of the homological dimensions. In particular, they satisfy an analog of the Auslander-Buchsbaum formula.
\end{abstract}

\section{INTRODUCTION}

Modules of finite classical (that is projective or injective) dimension over a local ring $R$ have rather special properties. A hypothesis on finiteness of a classical dimension imposes stringent restrictions on the structure of the underlying ring.

The best studied non-classical homological dimension is the Gorenstein dimension. In [1], Auslander and Bridger assign to each $R$-module $M$ a number denoted by $\mathrm{G}-\operatorname{dim}_{R} M$, and called the Gorenstein dimension, or G-dimension of $M$. It corresponds to the Gorenstein property of rings.

In [3], a new homological invariant for a finite module $M$, called the complete intersection dimension and denoted $C I-\operatorname{dim}_{R} M$ is defined, which reflects complete intersection property of rings. Its definition involves a classical dimension and a class of surjective ring homomorphisms with special kernels.

Modelled on this, some new dimensions have been defined. Veliche [8] introduces the upper Gorenstein dimension, $G^{*}$-dimension and Gerko [6] defines the Cohen-Macaulay dimension, CM-dimension. The class of surjective homomorphisms of these new dimensions is defined such that their kernels satisfy a notion of perfection. More precisely, in view of the known inequalities $\operatorname{grade}_{R} M \leqslant \mathrm{G}-\operatorname{dim}_{R} M \leqslant \operatorname{pd}_{R} M$, the notion of a perfect module, that is, a module $M$ satisfying $\operatorname{grade}_{R} M=\operatorname{pd}_{R} M$, and the notion of a G-perfect module, that is, module $M$ satisfying $\operatorname{grade}_{R} M=\mathrm{G}-\operatorname{dim}_{R} M$, was defined (G-perfect modules are introduced in [5] and studied further in [7]). Using these notions,

Received 15th December, 2004

This research was in part supported by a grant from IPM (No. 83130014 and No. 83130028).

Copyright Clearance Centre, Inc. Serial-fee code: 0004-9727/05 \$A2.00+0.00. 
in order to define $\mathrm{G}^{*}$-dimension and $\mathrm{CM}$-dimension, they defined the class of homomorphisms $Q \rightarrow R^{\prime}$ to be surjective homomorphisms with respectively perfect and G-perfect kernels. This plays a key role in their definitions.

So it is a natural try to define another homological dimension, introducing new notions of perfection. This is the main aim of this paper. To this end, first we show that $\mathrm{CM}-\operatorname{dim}_{R} M$ is greater than or equal to grade ${ }_{R} M$. So we get a sequence of inequalities

$$
\operatorname{grade}_{R} M \leqslant \mathrm{CM}-\operatorname{dim}_{R} M \leqslant \mathrm{G}-\operatorname{dim}_{R} M \leqslant \mathrm{pd}_{R} M .
$$

Hence one can introduce CM-perfect modules as those modules whose grade is equal to their CM-dimension. Using this, a new homological dimension can be defined as

$$
\inf \left\{\begin{array}{l|l}
\mathrm{CM}-\operatorname{dim}_{Q} M^{\prime}-\mathrm{CM}-\operatorname{dim}_{Q} R^{\prime} & \begin{array}{c}
R \rightarrow R^{\prime} \leftarrow Q \text { is a } \\
\mathrm{CM} \text {-quasi-deformation }
\end{array}
\end{array}\right\},
$$

where by a CM-quasi-deformation we mean a diagram of local homomorphisms $R \rightarrow R^{\prime} \leftarrow Q$, where $R \rightarrow R^{\prime}$ is flat and $Q \rightarrow R^{\prime}$ is a surjective homomorphism whose kernel is CM-perfect. We show that this dimension shares many basic properties with other homological dimensions. It localises, it satisfies an analogue of the AuslanderBuchsbaum formula, and it reflects the Cohen-Macaulay property of rings. Moreover it interpolates between the $\operatorname{Rfd}_{R}$ and CM-dimension, where $\operatorname{Rfd}_{R} M$ denotes the restricted flat dimension of $M$, defined in [4] as

$$
\operatorname{Rfd}_{R} M=\sup \left\{\operatorname{depth} R_{\mathfrak{p}}-\operatorname{depth}_{R_{\mathfrak{p}}} M_{\mathfrak{p}} \mid \mathfrak{p} \in \operatorname{Spec}(R)\right\} .
$$

So we call it lower Cohen-Macaulay dimension and denote it by $\mathrm{CM}_{*}$-dimension.

During the work we observed that, by the same argument we used for the proof of Proposition 2.1, another term can add to the above sequence of inequalities, that is,

$$
\operatorname{grade}_{R} M \leqslant \mathrm{CM}_{*}-\operatorname{dim}_{R} M \leqslant \mathrm{CM}-\operatorname{dim}_{R} M \leqslant \mathrm{G}-\operatorname{dim}_{R} M \leqslant \operatorname{pd}_{R} M .
$$

In fact the argument works for any dimension that localises and satisfies the AuslanderBuchsbaum depth formula. This means that any such dimension is greater than or equal to the grade. So, using the class of surjective homomorphisms $Q \rightarrow R^{\prime}$ such that $R^{\prime}$ satisfies the equality $\operatorname{grade}_{Q} R^{\prime}=\mathrm{CM}_{*}-\operatorname{dim}_{Q} R^{\prime}$ a new dimension can be defined. It can be easily seen that it is a homological dimension. In particular, it localises and satisfies Auslander-Buchsbaum formula. Moreover it is related to the Cohen-Macaulay property of the ring and interpolates between $\operatorname{Rfd}_{R} M$ and $\mathrm{CM}_{*}-\operatorname{dim}_{R} M$. This procedure can iterated and so a sequence of homological dimensions will be obtained.

The third section of the paper is devoted to defining an upper bound for CMdimension. It's definition is based on the upper Gorenstein dimension of Veliche. We show that it satisfies the basic properties of homological dimensions and corresponds to 
Cohen-Macaulay property of the ring. It will be denoted by $\mathrm{CM}^{*}$-dimension, and in the hierarchy of homological dimensions it satisfies

$$
\operatorname{Rfd}_{R} M \leqslant \mathrm{CM}_{*}-\operatorname{dim}_{R} M \leqslant \mathrm{CM}-\operatorname{dim}_{R} M \leqslant\left\{\begin{array}{c}
\mathrm{G}-\operatorname{dim}_{R} M \\
\mathrm{CM}^{*}-\operatorname{dim}_{R} M
\end{array}\right\} \leqslant \mathrm{G}^{*}-\operatorname{dim}_{R} M .
$$

Also we are able to obtain an increasing sequence of homological dimensions, all finer than $\mathrm{CM}^{*}$-dimension and greater than CM-dimension. All of them are correspond to the Cohen-Macaulay property of rings.

\section{Lower Cohen-Macaulay Dimension}

Throughout the paper, $R$ is a local ring with maximal ideal $m$ and residue field $k=R / \mathrm{m}$. All modules are finitely generated. $M$ always denotes a finitely generated $R$-module. Recall that the grade of $M$ was defined by Rees as the least integer $i \geqslant 0$ such that $\operatorname{Ext}_{R}^{i}(M, R) \neq 0$. When $I$ is an ideal of $R$, the grade of the $R$-module $R / I$ will be denoted by grade $(I, R)$.

The Cohen-Macaulay dimension of $M$ is introduced by Gerko [6], by the formula

$$
\mathrm{CM}-\operatorname{dim}_{R} M=\inf \left\{\begin{array}{l|l}
\mathrm{G}-\operatorname{dim}_{Q} M^{\prime}-\mathrm{G}-\operatorname{dim}_{Q} R^{\prime} & \begin{array}{c}
R \rightarrow R^{\prime} \leftarrow Q \text { is a } \\
\mathrm{G} \text {-quasi-deformation }
\end{array}
\end{array}\right\},
$$

where a G-quasi-deformation is a diagram of local homomorphisms $R \rightarrow R^{\prime} \leftarrow Q$ such that $R \rightarrow R^{\prime}$ is flat and $Q \rightarrow R^{\prime}$ is a surjective homomorphism whose kernel is G-perfect. It is finer than G-dimension and greater than restricted flat dimension.

Proposition 2.1. Let $M$ be a finite $R$-module. Then

$$
\operatorname{grade}_{R} M \leqslant \mathrm{CM}-\operatorname{dim}_{R} M .
$$

Proof: Without loss of generality we may assume that $\mathrm{CM}-\operatorname{dim}_{R} M$ is finite. Set $J=\operatorname{Ann}_{R} M$. Let $\mathfrak{p}$ be any minimal prime ideal of $J$. Then

$$
\operatorname{grade}_{R} M=\operatorname{grade}(J, R) \leqslant \operatorname{grade}(\mathfrak{p}, R) \leqslant \operatorname{depth} R_{\mathfrak{p}} .
$$

Moreover, by the choice of $\mathfrak{p}$, it is clear that depth $M_{\mathfrak{p}}=0$. Since by [6, 3.10], $\mathrm{CM}-\operatorname{dim}_{R_{\mathrm{p}}} M_{\mathfrak{p}}$ is less than or equal to $\mathrm{CM}-\operatorname{dim}_{R} M$, it is finite. Hence it follows from the Auslander-Buchsbaum depth formula that $\mathrm{CM}-\operatorname{dim}_{R_{\mathrm{p}}} M_{\mathrm{p}}=\operatorname{depth} R_{\mathrm{p}}$. Now the result follows from the inequalities

$$
\text { grade }_{R} M \leqslant \operatorname{depth} R_{\mathrm{p}}=\mathrm{CM}-\operatorname{dim}_{R_{\mathrm{p}}} M_{\mathrm{p}} \leqslant \mathrm{CM}-\operatorname{dim}_{R} M .
$$

The above theorem provides motivation for the following definition. 
Definition 2.2: Let $M$ be a finitely generated $R$-module. In view of the previous proposition, we have

$$
\operatorname{grade}_{R} M \leqslant \mathrm{CM}-\operatorname{dim}_{R} M \leqslant \mathrm{G}-\operatorname{dim}_{R} M .
$$

We call $M$ to be CM-perfect if

$$
\operatorname{grade}_{R} M=\mathrm{CM}-\operatorname{dim}_{R} M .
$$

Clearly every G-perfect module is CM-perfect. Let $Q$ be a local ring and $J$ be an ideal of $Q$. Abusing terminology, $J$ is called CM-perfect if the $Q$-module $Q / J$ is CM-perfect.

We say that $R$ has a CM-deformation if there exists a local ring $Q$ and a CM-perfect ideal $J$ in $Q$ such that $R=Q / J$. A CM-quasi-deformation of $R$ is a diagram of local homomorphisms $R \rightarrow R^{\prime} \leftarrow Q$ with $R \rightarrow R^{\prime}$ a flat extension and $Q \rightarrow R^{\prime}$ a CM-deformation. Set $M^{\prime}=M \bigotimes_{R} R^{\prime}$.

Lower Cohen-Macaulay dimension. For a module $M \neq 0$ over a local ring $R$, we define

$$
\mathrm{CM}_{*}-\operatorname{dim}_{R} M=\inf \left\{\begin{array}{l|l}
\mathrm{CM}-\operatorname{dim}_{Q} M^{\prime}-\mathrm{CM}-\operatorname{dim}_{Q} R^{\prime} & \begin{array}{c}
R \rightarrow R^{\prime} \leftarrow Q \text { is a } \\
\mathrm{CM} \text {-quasi-deformation }
\end{array}
\end{array}\right\}
$$

and complement this by $\mathrm{CM}-\operatorname{dim}_{R} 0=-\infty$.

The purpose of the next results is to show that $\mathrm{CM}_{*}$-dimension has properties similar to those of the other known homological dimensions. First we present a version of Auslander-Buchsbaum formula for $\mathrm{CM}_{*}$-dimension.

THEOREM 2.3. If $\mathrm{CM}_{*}-\operatorname{dim}_{R} M<\infty$, then

$$
\mathrm{CM}_{*}-\operatorname{dim}_{R} M=\operatorname{depth}_{R} R-\operatorname{depth}_{R} M .
$$

Proof: By assumption, there exists a CM-quasi-deformation $R \rightarrow R^{\prime} \leftarrow Q$ with $\mathrm{CM}-\operatorname{dim}_{Q} M^{\prime}<\infty$. Moreover $\mathrm{CM}_{*} \operatorname{dim}_{R} M=\mathrm{CM}-\operatorname{dim}_{Q} M^{\prime}-\mathrm{CM}-\operatorname{dim}_{Q} R^{\prime}$. Now the result follows from the faithful flatness of $R \rightarrow R^{\prime}$ and also Auslander-Buchsbaum formula for CM-dimension.

TheOREM 2.4. For any prime $\mathfrak{p} \in \operatorname{Supp}_{R} M$ there is an inequality

$$
\mathrm{CM}_{*}-\operatorname{dim}_{R_{\mathfrak{p}}} M_{\mathfrak{p}} \leqslant \mathrm{CM}_{*}-\operatorname{dim}_{R} M .
$$

Proof: It suffices to assume that $\mathrm{CM}_{*}-\operatorname{dim}_{R} M$ is finite. Let $R \rightarrow R^{\prime} \leftarrow Q$ be a CM-quasi-deformation with $\mathrm{CM}-\operatorname{dim}_{Q} M^{\prime}<\infty$. It follows from the faithful flatness of $R \rightarrow R^{\prime}$ that there exists a prime ideal $\mathfrak{p}^{\prime}$ in $R^{\prime}$ lying over $\mathfrak{p}$. Let $q$ be the inverse image of $\mathfrak{p}^{\prime}$ in $Q$. It follows from the inequalities

$$
\operatorname{grade}_{Q} R^{\prime} \leqslant \operatorname{grade}_{Q_{\mathrm{q}}} R_{\mathrm{q}}^{\prime} \leqslant \mathrm{CM}-\operatorname{dim}_{Q_{\mathrm{q}}} R_{\mathrm{q}}^{\prime} \leqslant \mathrm{CM}-\operatorname{dim}_{Q} R^{\prime},
$$


that $R_{\mathfrak{p}} \rightarrow R_{\mathfrak{p}^{\prime}}^{\prime} \leftarrow Q_{\mathrm{q}}$ is also a CM-quasi-deformation. Moreover we can conclude that $\mathrm{CM}-\operatorname{dim}_{Q_{\mathrm{q}}} R_{\mathrm{q}}^{\prime}=\mathrm{CM}-\operatorname{dim}_{Q} R^{\prime}$. Now the result follows using $[6,3.10]$.

The position of $\mathrm{CM}_{*}$-dimension in the hierarchy of homological dimensions is given by the following proposition.

Propos ItION 2.5. The following inequalities hold

$$
\operatorname{Rfd}_{R} M \leqslant \mathrm{CM}_{*}-\operatorname{dim}_{R} M \leqslant \mathrm{CM}-\operatorname{dim}_{R} M,
$$

with equality to the left of any finite term.

Proof: For the first inequality we must only deal the case where $\mathrm{CM}_{*}-\operatorname{dim}_{R} M$ is finite. By Theorem $2.3, \mathrm{CM}_{*}-\operatorname{dim}_{R} M=\operatorname{depth} R-\operatorname{depth}_{R} M$ and by Theorem 2.4, $\mathrm{CM}_{*}-\operatorname{dim}_{R_{\mathrm{p}}} M_{\mathrm{p}} \leqslant \mathrm{CM}_{*}-\operatorname{dim}_{R} M$, for all $\mathfrak{p} \in \operatorname{Spec} R$. So for a suitable prime $\mathfrak{p}$, we have $\operatorname{Rfd}_{R} M=\operatorname{depth} R_{\mathrm{p}}-\operatorname{depth}_{R_{\mathrm{p}}} M_{\mathfrak{p}}=\mathrm{CM}_{*}-\operatorname{dim}_{R_{\mathrm{p}}} M_{\mathfrak{p}}$. But

$$
\mathrm{CM}_{*}-\operatorname{dim}_{R_{\mathrm{p}}} M_{\mathrm{p}} \leqslant \mathrm{CM}_{*}-\operatorname{dim}_{R} M=\operatorname{depth} R-\operatorname{depth}_{R} M \leqslant \operatorname{Rfd}_{R} M .
$$

Hence $\mathrm{CM}_{*}-\operatorname{dim}_{R} M=\operatorname{Rfd}_{R} M$.

For the second inequality, again without loss of generality we may assume that $\mathrm{CM}-\operatorname{dim}_{R} M$ is finite. Now the inequality follows because every G-quasi-deformation is clearly a CM-quasi-deformation, and so appears in the determination of $\mathrm{CM}_{*}-\operatorname{dim}_{R} M$. The equality follows easily using the Auslander-Buchsbaum formula.

Note that with regard to the above proposition, when $\mathrm{CM}_{*}-\operatorname{dim}_{R} M$ is finite, the restricted flat dimension is achieved at the maximal ideal.

The next result provides a characterisation for Cohen-Macaulay rings in terms of the $\mathrm{CM}_{*}$-dimension of their modules.

THEOREM 2.6. The following are equivalent.

(i) $R$ is Cohen-Macaulay.

(ii) $\mathrm{CM}_{*}-\operatorname{dim}_{R} M<\infty$ for every finitely generated $R$-module $M$.

Proof: (i) $\Rightarrow$ (ii). This follows from [6, 3.9] and Proposition 2.4.

(ii) $\Rightarrow$ (i). Assume that $R$ is not Cohen-Macaulay. We seek a contradiction. Let $x_{1}, x_{2}, \cdots, x_{d}$ be a maximal $R$-sequence, where $d=\operatorname{depth} R$. By our assumption the ideal generated by this sequence $(\bar{x})$ can not be m-primary. So there exists a prime ideal $\mathfrak{p}$ such that $(\bar{x}) \subseteq \mathfrak{p} \subset \mathfrak{m}$. Consider the module $M=R / \mathfrak{p}$. Clearly $\operatorname{depth}_{R} M>0$. So $\operatorname{depth} R-\operatorname{depth}_{R} M<d$. But depth $R_{R_{\mathrm{p}}} M_{\mathrm{p}}=0$ and depth $R_{\mathrm{p}} \geqslant d$. Hence $d \leqslant \operatorname{depth}$ $R_{\mathfrak{p}}-\operatorname{depth}_{R_{\mathrm{p}}} M_{\mathfrak{p}}=\mathrm{CM}_{*}-\operatorname{dim}_{R_{\mathrm{p}}} M_{\mathrm{p}}$. But in view of the Theorem 2.4, this is a contradiction.

The $n$ 'th syzygy of a finite module $M$ over a local ring $R$ is defined uniquely up to isomorphism by $\operatorname{Syz}_{n}^{R}(M)=$ Coker $\partial_{n+1}$, where $(\mathbf{F}, \partial)$ is a minimal free resolution of $M$. 
If $R \rightarrow R^{\prime}$ is a local flat extension, then

$$
\operatorname{Syz}_{n}^{R^{\prime}}\left(M^{\prime}\right) \cong \operatorname{Syz}_{n}^{R}(M) \bigotimes_{R} R^{\prime}
$$

Proposition 2.7. If $M \neq 0$, for $n \geqslant 0$ there is an equality

$$
\mathrm{CM}_{*}-\operatorname{dim}_{R} \operatorname{Syz}_{n}^{R}(M)=\operatorname{Max}\left\{\mathrm{CM}_{*}-\operatorname{dim}_{R} M-n, 0\right\} .
$$

Proof: If $R \rightarrow R^{\prime} \leftarrow Q$ is a CM-quasi-deformation, then it is easily seen, that $\mathrm{CM}-\operatorname{dim}_{Q} \operatorname{Syz}_{n}^{R^{\prime}}\left(M^{\prime}\right)$ is finite if and only if $\mathrm{CM}-\operatorname{dim}_{Q} M^{\prime}$ is. So it follows that $\mathrm{CM}_{*}-\operatorname{dim}_{R} \operatorname{Syz}_{n}^{R}(M)$ is finite if and only if $\mathrm{CM}_{*}-\operatorname{dim}_{R} M$ is. Therefore we may assume that $\mathrm{CM}_{*}-\operatorname{dim}_{R} M<\infty$. By Theorem 2.3, it is enough to prove

$$
\operatorname{depth}_{R} \operatorname{Syz}_{n}^{R}(M)=\operatorname{Min}\left\{\operatorname{depth}_{R} M+n, \operatorname{depth} R\right\} \text { for } 0 \leqslant n \leqslant \operatorname{pd}_{R} M .
$$

We prove this using induction on $n$. By Theorem $2.3, \operatorname{depth}_{R} \operatorname{Syz}_{n}^{R}(M) \leqslant \operatorname{depth} R$ for all $0 \leqslant n \leqslant \operatorname{pd}_{R} M$.

The induction start, $n=0$ is trivial. The case $n=1$ follows from $[2,1.2 .6]$. The induction step now completes by another use of $[2,1.2 .6]$.

Proposition 2.8. Let $R \rightarrow S$ be a local flat extension. Then

$$
\mathrm{CM}_{*}-\operatorname{dim}_{R} M \leqslant \mathrm{CM}_{*}-\operatorname{dim}_{S}\left(M \bigotimes_{R} S\right)
$$

with equality when $\mathrm{CM}_{*}-\operatorname{dim}_{S}\left(M{\underset{R}{ } S}_{S}\right)$ is finite.

Proof: We may assume that $\mathrm{CM}_{*}-\operatorname{dim}_{S}\left(M \bigotimes_{R} S\right)$ is finite. Let $S \rightarrow R^{\prime} \leftarrow Q$ be the corresponding CM-quasi-deformation. Since $R \rightarrow S$ and $S \rightarrow R^{\prime}$ are flat extensions, the homomorphism $R \rightarrow R^{\prime}$ is also flat, so $R \rightarrow R^{\prime} \leftarrow Q$ is again a CM-quasi-deformation. This proves the inequality. To complete the proof remember the following equalities

$$
\begin{aligned}
\mathrm{CM}_{*}-\operatorname{dim}_{S}\left(M \bigotimes_{R} S\right) & =\operatorname{depth} S-\operatorname{depth}_{S}\left(M \bigotimes_{R} S\right) \\
& =\operatorname{depth} R-\operatorname{depth}_{R} M \\
& =\mathrm{CM}_{*}-\operatorname{dim}_{R} M .
\end{aligned}
$$

REMARK 2.9. Since the lower Cohen-Macaulay dimension localises and satisfies the Auslander-Buchsbaum formula, the argument we have used for the proof of Proposition 2.1 , can be applied to it. So we can conclude that $\operatorname{grade}_{R} M \leqslant \mathrm{CM}_{*}-\operatorname{dim}_{R} M$. This lead us to a new notion of perfection, say $\mathrm{CM}_{*}$-perfect modules. Using it, one can introduce $\mathrm{CM}_{*}$-quasi-deformation and use it for defining another homological dimension, 
say $\mathrm{CM}_{* *}$-dimension. All of the above results, are valid for $\mathrm{CM}_{* *}$-dimension. In particular, it localises and satisfies Auslander-Buchsbaum formula. Furthermore, it interpolates between restricted flat dimension and lower Cohen-Macaulay dimension. Hence another use of Proposition 2.1 shows that $\operatorname{grade}_{R} M \leqslant \mathrm{CM}_{* *}-\operatorname{dim}_{R} M$. Hence the above procedure can be iterated to define new dimensions. So we can record the following sequence of inequalities

$$
\operatorname{Rfd}_{R} M \leqslant \cdots \leqslant \mathrm{CM}_{* *}-\operatorname{dim}_{R} M \leqslant \mathrm{CM}_{*}-\operatorname{dim}_{R} M \leqslant \mathrm{CM}-\operatorname{dim}_{R} M \leqslant \mathrm{G}-\operatorname{dim}_{R} M .
$$

\section{Upper Cohen-Macaulay Dimension}

In [8], Veliche has defined a homological dimension, $\mathrm{G}^{*}$-dim, that corresponds to the Gorenstein property of rings. Let us recall her definition. A Gorenstein quasi-deformation of $R$ is a diagram of local homomorphisms $R \rightarrow R^{\prime} \leftarrow Q$, with $R \rightarrow R^{\prime}$ a flat extension and $R^{\prime} \leftarrow Q$ a surjective homomorphism whose kernel is a Gorenstein ideal, that is, it is perfect and $\beta_{g}^{Q}(Q / J)=1$ for $g=\operatorname{grade}(J, Q)$. As usual set $M^{\prime}=M \bigotimes_{R} R^{\prime}$. The upper Gorenstein dimension of the $R$-module $M$ is defined by the formula

$$
\mathrm{G}^{*}-\operatorname{dim}_{R} M=\inf \left\{\operatorname{pd}_{Q} M^{\prime}-\operatorname{pd}_{Q} R^{\prime} \mid \begin{array}{c}
R \rightarrow R^{\prime} \leftarrow Q \text { is a } \\
\text { Gorenstein quasi deformation }
\end{array}\right\} .
$$

It can be located in the following sequence of inequalities:

$$
\operatorname{grade}_{R} M \leqslant \mathrm{G}-\operatorname{dim}_{R} M \leqslant \mathrm{G}^{*}-\operatorname{dim}_{R} M \leqslant C I-\operatorname{dim}_{R} M .
$$

We define an $R$-module $M$ to be $\mathrm{G}^{*}$-perfect where $\operatorname{grade}_{R} M=\mathrm{G}^{*}-\operatorname{dim}_{R} M$. Clearly every $\mathrm{G}^{*}$-perfect module is G-perfect. Similarly an ideal $J$ is called $\mathrm{G}^{*}$-perfect if the $R$-module $R / J$ is $\mathrm{G}^{*}$-perfect.

A $\mathrm{G}^{*}$-deformation for $R$ is a surjective local homomorphism $Q \rightarrow R$ such that its kernel $J$, is $\mathrm{G}^{*}$-perfect. A $\mathrm{G}^{*}$-quasi-deformation for $R$ is a diagram of local homomorphisms $R \rightarrow R^{\prime} \leftarrow Q$, where $R \rightarrow R^{\prime}$ is a flat extension and $Q \rightarrow R^{\prime}$ is a $\mathrm{G}^{*}$-deformation. Let $M^{\prime}=M \bigotimes_{R} R^{\prime}$. We define the upper Cohen-Macaulay dimension of $M$ by

$$
\mathrm{CM}^{*}-\operatorname{dim}_{R} M=\inf \left\{\begin{array}{l|l}
\mathrm{G}^{*}-\operatorname{dim}_{Q} M^{\prime}-\mathrm{G}^{*}-\operatorname{dim}_{Q} R^{\prime} & \begin{array}{c}
R \rightarrow R^{\prime} \leftarrow Q \text { is a } \\
\mathrm{G}^{*} \text {-quasi-deformation }
\end{array}
\end{array}\right\}
$$

Since every $\mathrm{G}^{*}$-quasi-deformation is a G-quasi-deformation, it follows from the definition that

$$
\mathrm{CM}-\operatorname{dim}_{R} M \leqslant \mathrm{CM}^{*}-\operatorname{dim}_{R} M \leqslant \mathrm{G}^{*}-\operatorname{dim}_{R} M .
$$

The following results, which show that $\mathrm{CM}^{*}$-dimension is really a homological dimension with the expected properties, can be proved using exactly the same arguments as we used in the previous section for proving the similar results. So we omit most of their proofs. 
ThEOREM 3.1. If $\mathrm{CM}^{*}-\operatorname{dim}_{R} M<\infty$, then

$$
\mathrm{CM}^{*}-\operatorname{dim}_{R} M=\operatorname{depth} R-\operatorname{depth}_{R} M .
$$

Proposition 3.2 . If $M \neq 0$, for $n \geqslant 0$

$$
\mathrm{CM}^{*}-\operatorname{dim}_{R} \operatorname{Syz}_{n}^{R}(M)=\operatorname{Max}\left\{\mathrm{CM}^{*}-\operatorname{dim}_{R} M-n, 0\right\} .
$$

THEOREM 3.3. The following are equivalent.

(i) $R$ is Cohen-Macaulay.

(ii) $\mathrm{CM}^{*}-\operatorname{dim}_{R} M<\infty$ for every finitely generated $R$-module $M$.

(iii) $\mathrm{CM}^{*}-\operatorname{dim}_{R} k=\operatorname{depth} R$.

(iv) $\mathrm{CM}^{*}-\operatorname{dim}_{R} k<\infty$.

Proof: (i) $\Rightarrow$ (ii). Let $\widehat{R}$ be the completion of $R$ with respect to maximal ideal m. Then $\widehat{R}$ is Cohen-Macaulay and by Cohen's structure theorem, can be written as a quotient of a regular ring $Q$ with kernel, say $J$. It is easy to see that $J$ is $\mathrm{G}^{*}$-perfect. So $R \rightarrow R^{\prime} \leftarrow Q$ is a $\mathrm{G}^{*}$-quasi-deformation. Now the finiteness of $\mathrm{CM}^{*}$-dimension follows from the fact that over regular ring $Q$, every module has finite $\mathrm{G}^{*}$-dimension.

(ii) $\Rightarrow$ (iii). Follows from 3.1.

(iii) $\Rightarrow$ (iv). This holds trivally.

(iv) $\Rightarrow$ (i). If $\mathrm{CM}^{*}-\operatorname{dim}_{R} k<\infty$, then $\mathrm{CM}-\operatorname{dim}_{R} k$ is finite and so by $[6,3.9], R$ is Cohen-Macaulay.

Proposition 3.4. If $R \rightarrow S$ is a local flat extension, then

$$
\mathrm{CM}^{*}-\operatorname{dim}_{R} M \leqslant \mathrm{CM}^{*}-\operatorname{dim}_{S}\left(M \bigotimes_{R} S\right)
$$

with equality when $\mathrm{CM}^{*}-\operatorname{dim}_{S}\left(M \bigotimes_{R} S\right)$ is finite.

Proposition 3.5. For any prime $\mathfrak{p} \in \operatorname{Supp}_{R} M$,

$$
\mathrm{CM}^{*}-\operatorname{dim}_{R_{\mathrm{p}}} M_{\mathrm{p}} \leqslant \mathrm{CM}^{*}-\operatorname{dim}_{R} M
$$

Proof: Suppose CM* $-\operatorname{dim}_{R} M<\infty$ and $R \rightarrow R^{\prime} \leftarrow Q$ is the corresponding $\mathrm{G}^{*}-$ quasi-deformation, with $R^{\prime}=Q / J$. Let $\mathfrak{p}^{\prime} \in \operatorname{Spec}\left(R^{\prime}\right)$ be such that $R \cap \mathfrak{p}^{\prime}=\mathfrak{p}$ and $\mathfrak{q} \subseteq Q$ be the pre-image of $\mathfrak{p}^{\prime}$. In view of $[8,2.10]$, the inequalities

$$
\text { grade }(J, Q) \leqslant \operatorname{grade}\left(J_{\mathfrak{q}}, Q_{q}\right) \leqslant \mathrm{G}^{*}-\operatorname{dim}_{Q_{\mathfrak{q}}} Q_{\mathfrak{q}} / J_{\mathfrak{q}} \leqslant \mathrm{G}^{*}-\operatorname{dim}_{Q} Q / J
$$

hold. Since $J$ is $\mathrm{G}^{*}$-perfect, they become equalities and so $J_{\mathfrak{q}}$ is $\mathrm{G}^{*}$-perfect. Hence $R_{\mathrm{p}} \rightarrow R_{\mathfrak{p}^{\prime}}^{\prime} \leftarrow Q_{\mathfrak{q}}$ is a $G^{*}$-quasi-deformation. Now the proof can be completed easily. 
REMARK 3.6. It is clear that for an $R$-module $M, \mathrm{CM}^{*}-\operatorname{dim}_{R} M$ is greater than grade $_{R} M$. So we can introduce an $R$-module $M$, with the property that grade ${ }_{R}$ $M=\mathrm{CM}^{*}-\operatorname{dim}_{R} M$, to be $\mathrm{CM}^{*}$-perfect. Similarly we can define $\mathrm{CM}^{*}$-quasi-deformation, as a diagram $R \rightarrow R^{\prime} \leftarrow Q$ of local homomorphism, with $R \rightarrow R^{\prime}$ flat and $Q \rightarrow R^{\prime}$ a surjective homomorphism making $R^{\prime}$ into a $\mathrm{CM}^{*}$-perfect $Q$-module. This means that a dimension can be introduced as

$$
\inf \left\{\begin{array}{l|l}
\mathrm{CM}^{*}-\operatorname{dim}_{Q} M^{\prime}-\mathrm{CM}^{*}-\operatorname{dim}_{Q} R^{\prime} & \begin{array}{c}
R \rightarrow R^{\prime} \leftarrow Q \text { is a } \\
\mathrm{CM}^{*} \text {-quasi-deformation }
\end{array}
\end{array}\right\} .
$$

We leave it to the reader to check, using similar arguments, that all the expected properties, parallel to those of the homological dimensions, are valid for this new dimension. If we denote this later dimension by $\mathrm{CM}^{* *}$-dim, we obtain a sequence of inequalities

$$
\mathrm{CM}-\operatorname{dim}_{R} M \leqslant \mathrm{CM}^{* *}-\operatorname{dim}_{R} M \leqslant \mathrm{CM}^{*}-\operatorname{dim}_{R} M \leqslant \mathrm{G}^{*}-\operatorname{dim}_{R} M .
$$

So following the above argument, we can introduce $\mathrm{CM}^{* *}$-perfect modules and so another homological dimension can be obtained. Therefore we have the following sequence of inequalities

$$
\mathrm{CM}-\operatorname{dim}_{R} M \leqslant \cdots \leqslant \mathrm{CM}^{* *}-\operatorname{dim}_{R} M \leqslant \mathrm{CM}^{*}-\operatorname{dim}_{R} M \leqslant \mathrm{G}^{*}-\operatorname{dim}_{R} M .
$$

Summing up the results in sections two and three, we can state the sequence of inequalities

$$
\begin{array}{r}
\operatorname{Rfd}_{R} M \leqslant \cdots \leqslant \mathrm{CM}_{*}-\operatorname{dim}_{R} M \leqslant \mathrm{CM}-\operatorname{dim}_{R} M \\
\leqslant \cdots \leqslant \mathrm{CM}^{*}-\operatorname{dim}_{R} M \leqslant \mathrm{G}^{*}-\operatorname{dim}_{R} M .
\end{array}
$$

Note that all the middle terms, reflect the Cohen-Macaulay property of rings. Actually we don't know if the displayed inequalities can be strict.

\section{REFERENCES}

[1] M. Auslander and M. Bridger, 'Stable module theory', Mem. Amer. Math. Soc. 94 (1969).

[2] L.L. Avramov, 'Infinite free resolutions', in Six lectures on commutative algebra (Bellaterra, 1996), Progr. Math. Vol. 166 (Birkhäuser, Basel, 1998), pp. 1-118.

[3] L.L. Avramov, V.N. Gasharov and IV. Peeva, 'Complete intersection dimension', Inst. Hautes Études Sci. Publ. Math. 86 (1997), 67-114.

[4] L.W. Christensen, H.-B. Foxby and A. Frankild, 'Restricted homological dimensions and Cohen-Macaulayness', J. Algebra 251 (2002), 479-502.

[5] H.-B. Foxby, 'Quasi-perfect modules over Cohen-Macaulay rings', Math. Nachr. 66 (1975), 103-110.

[6] A.A. Gerko, 'On homological dimensions', Sb. Math. 192 (2001), 1165-1179. 
[7] E.S. Golod, 'G-dimension and generalized perfect ideals', Trudy Mat. Inst. Stelov. 165 (1985), 67-71.

[8] O. Veliche, 'Construction of modules with finite homological dimensions', J. Algebra 250 (2002), 427-449.

Institute for Studies in Theoretical

Physics andMathematics

P.O.Box 19395-5746

Tehran

Iran

and

Faculty of Science

Shahre-Kord University

P.O.Box 115

Shahre-Kord

Iran

e-mail: Asadollahi@ipm.ir

\author{
School of Mathematics \\ Institute for studies in Theoretical \\ Physics and Mathematics (IPM) \\ P.O.Box: 19395-5746 \\ Tehran \\ Iran \\ and \\ Department of Mathematics \\ University of Isfahan \\ P.O. Box 81746-73441 \\ Isfahan \\ Iran \\ e-mail: Salarian@ipm.ir
}

